\title{
ARTICLE RepSox, a small molecule inhibitor of the TGF $\beta$ receptor, induces brown adipogenesis and browning of white adipocytes
}

Wan-zhi Tu${ }^{1,2,3}$, Yan-bin $\mathrm{Fu}^{1,4}$ and $\mathrm{Xin} \mathrm{Xie}^{1,2,3,4}$

Unlike white adipose tissue (WAT), brown adipose tissue (BAT) is mainly responsible for energy expenditure via thermogenesis by uncoupling the respiratory chain. Promoting the differentiation of brown fat precursor cells and the browning of white fat have become a research hotspot for the treatment of obesity and associated metabolic diseases. Several secreted factors and a number of small molecules have been found to promote brown adipogenesis. Here we report that a single small-molecule compound, RepSox, is sufficient to induce adipogenesis from mouse embryonic fibroblasts (MEFs) in fibroblast culture medium. RepSox is an inhibitor of the transforming growth factor-beta receptor I (TGF- $\beta$-RI), other inhibitors of TGF- $\beta$ pathway such as SB431542, LY2157299, A83-01, and Tranilast are also effective in inducing adipogenesis from MEFs. These adipocytes express brown adipocyte-specific transcription factors and thermogenesis genes, and contain a large number of mitochondria and have a high level of mitochondrial respiratory activity. More interestingly, RepSox has also been found to promote the differentiation of the brown fat precursor cells and induce browning of the white fat precursor cells. These findings suggest that inhibitors of TGF- $\beta$ signaling pathway might be developed as new therapeutics for obesity and type 2 diabetes.

Keywords: RepSox; brown adipogenesis; TGF- $\beta$ receptor; TGF- $\beta$ inhibitor; adipocyte; differentiation

Acta Pharmacologica Sinica (2019) 40:1523-1531; https://doi.org/10.1038/s41401-019-0264-2

\section{INTRODUCTION}

Obesity has become a worldwide epidemic and may lead to many other diseases, such as type 2 diabetes, cardiovascular diseases, and several types of cancers, which diminish life quality and life expectancy [1]. There are two types of adipose tissues in mammals: white adipose tissue (WAT) and brown adipose tissue (BAT) [2]. WAT is responsible for energy storage and is the primary adipose tissue in the mammalian body. Obesity is typically caused by more energy intake than expenditure and excessive accumulation of WAT. In contrast, BAT is mainly responsible for the consumption of energy and nonshivering thermogenesis in response to cold exposure.

Brown adipocytes contain abundant mitochondria and mitochondrial membrane uncoupling protein 1 (UCP1), which mediates heat generation by uncoupling the respiratory chain, allowing for fast glucose and fatty acid oxidation with a low efficiency of ATP production [3,4]. Although newborn mammals have considerable BAT in their bodies, adults have much less BAT. Previous studies have shown that after long-term cold stimulation or adrenaline treatment, WAT contains scattered brown-like adipocytes (beige adipocytes) [5-8], indicating a conversion between white and brown adipocytes.

Because of the energy consumption characteristics of brown adipocytes, promoting the differentiation of brown fat precursor cells and the browning of white adipocytes have become potential treatment strategies to battle obesity and type 2 diabetes [8]. Several secreted factors, including irisin, apelin, bone morphogenetic protein 7 (BMP7), and a number of small molecules, such as rosiglitazone (a peroxisome proliferatoractivated receptor gamma (PPAR $\gamma$ ) agonist), tofacitinib (a Janus kinase (JAK) inhibitor), and R406 (a spleen tyrosine kinase (SYK) inhibitor), have been found to promote brown adipogenesis [9-13]. Here, we report that RepSox, a small-molecule inhibitor of transforming growth factor-beta receptor I (TGF- $\beta$-RI) [14, 15], induces brown adipogenesis in primary mouse fibroblasts and fat precursor cells.

\section{MATERIALS AND METHODS}

Cell culture and differentiation

Mouse embryonic fibroblasts (MEFs) were isolated from embryonic day 13.5 (E13.5) C57BL/6 mouse embryos. The head, limbs, and internal organs were removed from the embryos. The remaining carcasses were rinsed with phosphate-buffered saline (PBS) and cut into small pieces. The minced carcasses were trypsinized into single-cell suspensions. MEFs were cultured in growth medium consisting of Dulbecco's modified Eagle's medium (DMEM) (Gibco, USA), 10\% fetal bovine serum (FBS) (Gibco, USA), 2 mM Glutamax (Gibco, USA), 100 units $/ \mathrm{mL}$ penicillin, and $100 \mathrm{mg} / \mathrm{mL}$ streptomycin at $37^{\circ} \mathrm{C}$ in a $5 \% \mathrm{CO}_{2}$ incubator. For differentiation, sufficiently confluent cells were maintained in differentiation induction medium containing various compounds

\footnotetext{
${ }^{1}$ CAS Key Laboratory of Receptor Research, The National Center for Drug Screening, Shanghai Institute of Materia Medica, Chinese Academy of Sciences, 201203 Shanghai, China; ${ }^{2}$ University of Chinese Academy of Sciences, 100049 Beijing, China; ${ }^{3}$ School of Life Science and Technology, ShanghaiTech University, 201210 Shanghai, China and ${ }^{4}$ Shanghai Key Laboratory of Signaling and Disease Research, Laboratory of Receptor-based Bio-medicine, School of Life Sciences and Technology, Tongji University, 200092 Shanghai, China Correspondence: Xin Xie (xxie@simm.ac.cn)
}

Received: 22 January 2019 Accepted: 27 May 2019

Published online: 24 June 2019 
for 8-10 days before further analysis. The medium was changed every 2 days.

To isolate primary preadipocytes, interscapular BAT and subcutaneous WAT were obtained from 5- to 6-week-old C57BL/ 6 mice. The tissues were minced with scissors and digested in dispase solution ( $1 \mathrm{U} / \mathrm{mL}$ in DMEM/F-12; STEMCELL, Canada) containing collagenase type IV $(10 \mathrm{mg} / \mathrm{mL}$; Gibco, USA) and $\mathrm{CaCl}_{2}(10 \mathrm{mM})$ at $37^{\circ} \mathrm{C}$ in a water bath shaker. After $45 \mathrm{~min}$, the contents were filtered through $70 \mu \mathrm{m}$ nylon strainers (BD Falcon, USA) to remove undigested parts, followed by centrifugation $(500 \times g$ for $10 \mathrm{~min})$. Thereafter, the infranatants were resuspended and filtered through $40 \mu \mathrm{m}$ nylon strainers (BD Falcon, USA) and centrifuged at $500 \times g$ for 5 min. Finally, the cells were plated and cultured in growth medium consisting of DMEM/F-12 (Gibco, USA), 10\% FBS, 100 units $/ \mathrm{mL}$ penicillin, and $100 \mu \mathrm{g} / \mathrm{mL}$ streptomycin at $37{ }^{\circ} \mathrm{C}$ in a $5 \% \mathrm{CO}_{2}$ incubator. Differentiation was induced by treating confluent cells with differentiation induction medium containing $0.5 \mathrm{mM}$ isobutylmethylxanthine (IBMX), $1 \mu \mathrm{M}$ dexamethasone, and $1 \mu \mathrm{g} / \mathrm{mL}$ insulin (plus $4 \mu \mathrm{g} / \mathrm{mL}$ insulin, $125 \mathrm{nM}$ indomethacin, and $1 \mathrm{nM} 3,5,3$ '-triiodothyronine (T3) for brown adipocyte differentiation) in growth medium during days $0-2$. At day 2 , the cells were moved to maintenance medium containing $1 \mu \mathrm{g} / \mathrm{mL}$ insulin (plus $4 \mu \mathrm{g} / \mathrm{mL}$ insulin and $1 \mathrm{nM}$ T3 for brown adipocyte differentiation) in growth medium for another 2 days. On days 4-8, the cells were maintained in growth medium. Test compounds, including RepSox, other TGF $\beta$ inhibitors, and rosiglitazone, were present throughout the whole differentiation process (days $0-8$ ). The medium was changed every 2 days.

\section{Oil red $O$ staining}

Differentiated cells were washed in PBS and then fixed in $4 \%$ paraformaldehyde for $20 \mathrm{~min}$ at room temperature. A mixture of oil red $\mathrm{O}$ solution $(0.5 \%$ oil red $\mathrm{O}$ dye in isopropanol) and water at a 3:2 ratio was layered onto the cells for $10 \mathrm{~min}$. After staining, excess oil red $\mathrm{O}$ was washed with $60 \%$ isopropanol, followed by washing with deionized water, and images were captured under a microscope.

\section{Triglyceride quantification assay}

The triglyceride concentration in adipocytes was measured using a triglyceride quantification assay kit (Abcam, USA) according to the manufacturer's protocol. The cells were digested with $0.25 \%$ trypsin and washed with cold PBS. The samples were resuspended in $1 \%$ Triton $\mathrm{X}-100$ and heated to $80-100^{\circ} \mathrm{C}$ for 2-5 min to solubilize triglycerides. Triglycerides were converted to glycerol and fatty acids by lipase. Glycerol was then oxidized to generate a product that reacts with a probe to generate color (spectrophotometry at $\lambda=570 \mathrm{~nm}$ ). The triglyceride concentrations in the samples were calculated by a standard curve.

\section{Western blot analysis}

Differentiated cells were washed with PBS, lysed, and boiled at $95-100{ }^{\circ} \mathrm{C}$ for $5 \mathrm{~min}$ in sample buffer $(50 \mathrm{mM}$ Tris- $\mathrm{HCl}, 2 \% \mathrm{w} / \mathrm{v}$ sodium dodecyl sulfate (SDS), $10 \%$ glycerol, $1 \% \beta$-mercaptoethanol, $0.01 \%$ bromophenyl blue ( $\mathrm{pH} 6.8)$ ). The protein lysates were separated through SDS-PAGE before being transferred to PVDF membranes. The membranes were first blocked in TBST (TBS with $0.1 \%$ Tween 20 ) containing $5 \%$ nonfat milk for $1 \mathrm{~h}$ at room temperature and then incubated overnight at $4{ }^{\circ} \mathrm{C}$ in TBST buffer containing mouse anti- $\beta$-tubulin (1:6000; Abmart, China) or rabbit anti-UCP1 (1:500; Alpha Diagnostic International, USA). The membranes were washed three times with TBST and incubated with anti-rabbit IgG HRP (1:8000; Cell Signaling, USA) or anti-mouse IgG HRP (1:8000; Abmart, China) for $1 \mathrm{~h}$ at room temperature. After washing three times with TBST, immunostaining was visualized using Western blotting detection reagents (GE Healthcare, USA).
Immunofluorescence and MitoTracker staining

To reveal the mitochondrial membrane potential and morphology, adipocytes were incubated with growth medium containing $150 \mathrm{nM}$ MitoTracker Deep Red FM (Invitrogen, USA) at $37^{\circ} \mathrm{C}$ in a $5 \% \mathrm{CO}_{2}$ incubator for $30 \mathrm{~min}$. Nuclei were counterstained with Hoechst 33342. Images were captured and analyzed with an Operetta High Content Imaging System.

For immunofluorescence staining, the cells were fixed in $4 \%$ PFA at room temperature for $20 \mathrm{~min}$ and washed twice with PBS. After permeabilizing with $0.3 \%$ Triton X-100 and blocking with $5 \%$ bovine serum albumin for $20 \mathrm{~min}$, the cells were incubated with a primary rabbit anti-UCP1 antibody (1:200; Alpha Diagnostic International, USA) at $4{ }^{\circ} \mathrm{C}$ overnight. After washing thoroughly, the cells were incubated with a secondary antibody conjugated to Alexa Fluor 488 (Molecular Probes, USA) for $1 \mathrm{~h}$ at room temperature, followed by washing three times with PBS. Nuclei were counterstained with Hoechst 33342 (Sigma, USA). Images were captured and analyzed with an Operetta High Content Imaging System.

\section{Oxygen consumption rate assay}

The oxygen consumption rates of differentiated cells were measured in a Seahorse XF96 Analyzer (Seahorse Bioscience Inc., USA) according to the manufacturer's protocol. Oxygen consumption rates were measured under basal conditions and after the addition of oligomycin $(1 \mu \mathrm{M})$, FCCP $(1 \mu \mathrm{M})$ and rotenone/antimycin $A(1 \mu M)$.

Quantitative real-time PCR analysis

Total mRNA was isolated using TRIzol (Invitrogen, USA), and $2 \mu \mathrm{g}$ of RNA was used to synthesize cDNA using the PrimeScript ${ }^{T M}$ RT Master Mix reagent kit (Takara, Japan) according to the manufacturer's protocol. Real-time PCR was performed using $2 \times$ SYBR Green qPCR Master Mix (Biotool, USA) and a Stratagene Mx 3000P thermal cycler. Real-time PCR primer sequences are listed in Table 1.

Statistical analyses

All data are presented as the mean \pm SEM, and the between-group differences were calculated with Student's $t$-test. All comparisons were two-tailed, and $P<0.05$ was considered statistically significant. All graphs were plotted with GraphPad Prism software.

\section{RESULTS}

TGF- $\beta$ receptor inhibitors induce adipogenesis in MEFs

In a previous study, we reported that cardiomyocytes could be induced from MEFs by the chemical cocktail CRFVPT (C, CHIR99021; R, RepSox, 616452; F, Forskolin; V, VPA; P, Parnate and $T, T T N P B)$ [16]. We intended to minimize this chemical cocktail, so various combinations of these compounds were tested to determine whether fewer compounds could be used to induce cardiomyocytes from MEFs. Unexpectedly, in the wells in which only CRF were used, a large number of adipocyte-like cells emerged (Fig. 1a).

Next, we sought to determine which of these compounds was critical in inducing adipogenesis in MEFs by using only one compound from the CRF set. Many adipocyte-like cells emerged in MEFs treated with RepSox, and oil red $O$ staining for lipid droplets confirmed adipocyte differentiation (Fig. 1b). Forskolin treatment also induced weak adipocyte differentiation, while treatment with CHIR99021 or DMSO had no effect (Fig. 1b). It seems that one small-molecule compound, RepSox, is sufficient for the induction of adipocytes from MEFs.

RepSox is a selective inhibitor of the TGF- $\beta$-RI/ALK5. Therefore, we investigated whether other inhibitors of the TGF- $\beta$ signaling pathway could also induce adipogenesis. MEFs were treated with SB431542, LY2157299, or A83-01, all TGF- $\beta$-RI/ALK5 inhibitors, 
Table 1. Real-Time PCR primer sequences

\begin{tabular}{|c|c|}
\hline Primer name & Sequence $\left(5^{\prime}-3^{\prime}\right)$ \\
\hline UCP1 F & AGGCTTCCAGTACCATTAGGT \\
\hline UCP1 R & CTGAGTGAGGCAAAGCTGATTT \\
\hline $\mathrm{PGC} 1 \alpha \mathrm{F}$ & TATGGAGTGACATAGAGTGTGCT \\
\hline PGC $1 \alpha R$ & CCACTTCAATCCACCCAGAAAG \\
\hline PRDM16 F & CCCCACATTCCGCTGTGAT \\
\hline PRDM16 R & CTCGCAATCCTTGCACTCA \\
\hline COX IV F & ATTGGCAAGAGAGCCATTTCTAC \\
\hline COX IV R & CACGCCGATCAGCGTAAGT \\
\hline $\mathrm{aP2} F$ & AAGGTGAAGAGCATCATAACCCT \\
\hline $\mathrm{aP2} \mathrm{R}$ & TCACGCCTTTCATAACACATTCC \\
\hline CEBP $\alpha F$ & CAAGAACAGCAACGAGTACCG \\
\hline CEBP $\alpha \mathrm{R}$ & GTCACTGGTCAACTCCAGCAC \\
\hline CEBP $\beta \mathrm{F}$ & GGGGTTGTTGATGTTTTTTGG \\
\hline CEBP $\beta \mathrm{R}$ & CGAAACGGAAAAGGTTCTCA \\
\hline CEBPS F & CGACTTCAGCGCCTACATTGA \\
\hline CEBPS R & GAAGAGGTCGGCGAAGAGTT \\
\hline PPAR $\gamma \mathrm{F}$ & TGTGGACCTCTCCGTGATGG \\
\hline PPAR $\gamma \mathrm{R}$ & GGTTCTACTTTGATCGCACTTTGG \\
\hline MCAD F & CCGAAGAGTTGGCGTATGGG \\
\hline MCAD R & GGGCTCTGTCACACAGTAAGC \\
\hline LCAD F & TTTCCTCGGAGCATGACATTTT \\
\hline LCAD R & GCCAGCTTTTTCCCAGACCT \\
\hline CPT2 F & CCTGCTCGCTCAGGATAAACA \\
\hline CPT2 R & GTGTCTTCAGAAACCGCACTG \\
\hline$\beta$-actin F & GGCTGTATTCCCCTCCATCG \\
\hline$\beta$-actin R & CCAGTTGGTAACAATGCCATGT \\
\hline
\end{tabular}

or Tranilast, a compound that inhibits TGF- $\beta$ secretion [17-20], and all these compounds were found to induce adipogenesis in MEFs, as confirmed by oil red $\mathrm{O}$ staining (Fig. 1c). These results indicate that blocking TGF- $\beta$ signaling is critical for inducing adipogenesis.

RepSox induces brown adipocytes from MEFs

RepSox-induced adipogenesis in MEFs was further investigated. The dose-response of RepSox was tested, and $3 \mu \mathrm{M}$ was found to be the most effective concentration (Fig. 2a). Therefore, $3 \mu \mathrm{M}$ RepSox was applied in the following experiments. Rosiglitazone, a PPAR $\gamma$ agonist, has been reported to promote adipogenesis and browning of white adipocytes [11]. We found that rosiglitazone $(1 \mu \mathrm{M})$ could enhance the adipogenesis efficiency of RepSox $(3 \mu \mathrm{M})$; however, rosiglitazone itself could not induce any adipocytes from MEFs at the tested concentration. Triglycerides within the cells were also quantified (Fig. 2b), and the results confirmed the findings obtained with oil red $\mathrm{O}$ staining.

RepSox treatment induced the activation of a network of genes controlling adipogenesis, energy expenditure, and the thermogenic program in MEFs (Fig. 2c). The expression levels of Ppary and CCAAT/enhancer-binding protein alpha (C/ebpa), which are the major transcription factors that control adipocyte differentiation [21], and adipocyte protein 2 (Ap2), a carrier protein for fatty acids in adipocytes, were strongly induced by RepSox treatment. CCAAT/enhancer-binding protein delta $(C / e b p \delta)$ also participate in the transcriptional cascade of adipogenesis. A previous study showed that the upregulation of $C / e b p \delta$ reached a maximal level during the first two days of differentiation and declined sharply to nearly undetectable levels during the final days of differentiation, with levels even lower than those of the starting adipoblasts [22]. We also found that at day 8 of differentiation, RepSox-treated cells had significantly lower levels of C/ebp $\delta$. RepSox also significantly induced the expression of brown adipocyte-specific genes, including Ucp1, Prdm16 (PR domain containing 16), Pgcla (PPARy coactivator 1 alpha), Coxiv (cytochrome $c$ oxidase subunit 4), and C/ebp $\beta$ (CCAAT/enhancer-binding protein beta). The expression of genes controlling fatty acid oxidation (FAO), such as Mcad (medium-chain acyl-CoA dehydrogenase), Lcad (long-chain acylCoA dehydrogenase), and Cpt2 (carnitine palmitoyltransferase 2), was enhanced by RepSox. Western blot analysis also confirmed the upregulation of UCP1 protein levels by RepSox treatment (Fig. 2d). These data suggest that RepSox alone is sufficient to induce brown adipocyte characteristics in MEFs, although the effect can be enhanced when combined with rosiglitazone.

Beige and brown adipocytes typically have an elevated number of mitochondria, which is essential for the thermogenic activity of BAT. MitoTracker Deep Red staining showed that RepSox treatment increased the number of mitochondria in MEF-derived adipocytes (Fig. 2e). The fluorescence intensity of the MitoTracker in the RepSox group was 1.5-fold higher than that of the control group, and combination with rosiglitazone further enhanced this effect (Fig. 2f). The expression of the mitochondria uncoupling protein UCP1 was further confirmed by immunocytochemical staining. The DMSO- and rosiglitazone-treated groups expressed very low levels of UCP1, but RepSox treatment alone or in combination with rosiglitazone significantly increased UCP1 levels (Fig. 2e, g). The oxygen consumption rate (OCR) of the MEFderived adipocytes was also measured. The basal, uncoupled and maximal respiration levels in the RepSox-treated cells were significantly increased compared with those in the DMSO control cells (Fig. $2 \mathrm{~h}$ ). These results suggest that RepSox might be a more powerful adipogenesis and browning inducer than rosiglitazone.

RepSox enhances brown preadipocyte differentiation

Brown preadipocytes were isolated by digesting the stromal vascular fraction (SVF) from the interscapular BAT [23]. The brown preadipocytes were induced to differentiate in MDIIT3 medium (DMEM/F-12 containing 10\% FBS, IBMX, dexamethasone, insulin, indomethacin, and T3, a medium typically used to induce brown adipogenesis) supplemented with RepSox, rosiglitazone, or their combination. Oil red $\mathrm{O}$ staining (Fig. 3a) and a triglyceride quantification assay (Fig. 3b) showed that both RepSox and rosiglitazone enhanced the differentiation of brown preadipocytes, and their combination had an even better effect. The mRNA levels of adipogenesis, brown adipogenesis, and FAO markers were significantly upregulated in cells treated with RepSox, rosiglitazone, or their combination (Fig. 3c). Western blot analysis also confirmed the increased UCP1 protein level in cells treated with RepSox or the combination of RepSox and rosiglitazone (Fig. 3d). MitoTracker Deep Red and UCP1 staining also demonstrated that RepSox significantly increased the number of mitochondria and the protein levels of UCP1, hence increasing the differentiation of brown preadipocytes (Fig. 3e-g). The OCR of the cells differentiated from brown preadipocytes was also significantly increased after RepSox treatment (Fig. 3h). The data show that RepSox can promote the differentiation of brown preadipocytes.

RepSox promotes browning of white preadipocytes Although RepSox could enhance brown characteristics when inducing adipogenesis from MEFs and promote the differentiation of brown preadipocytes, we wondered whether it could also promote the browning of primary white preadipocytes. White preadipocytes were isolated from subcutaneous WAT and induced to differentiate in MDI medium, which is typically used to induce white adipogenesis (DMEM/F-12 containing 10\% FBS, IBMX, dexamethasone, and insulin), supplemented with RepSox, rosiglitazone, or their combination. Oil red $\mathrm{O}$ staining and triglyceride quantification assays showed that both RepSox and rosiglitazone 
a

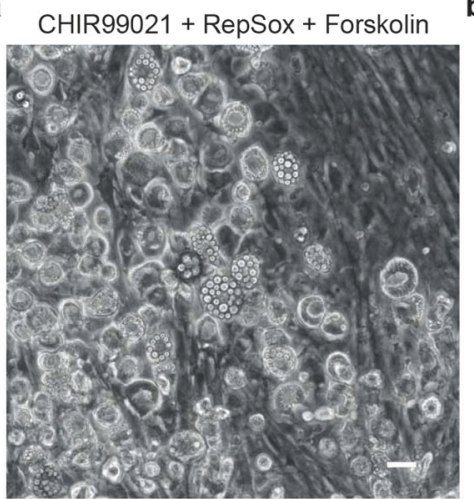

b

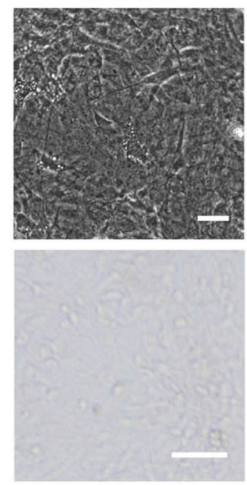

CHIR99021

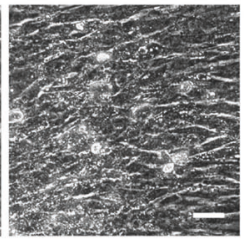

RepSox
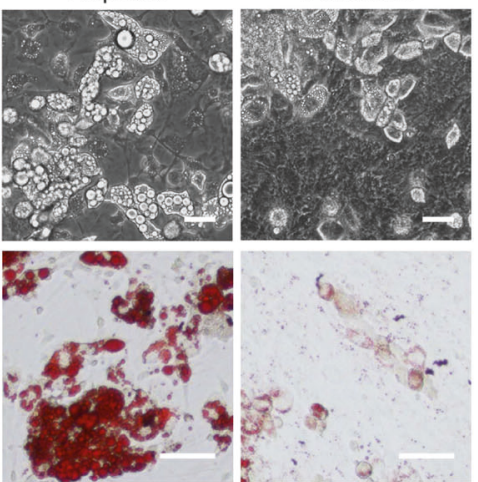
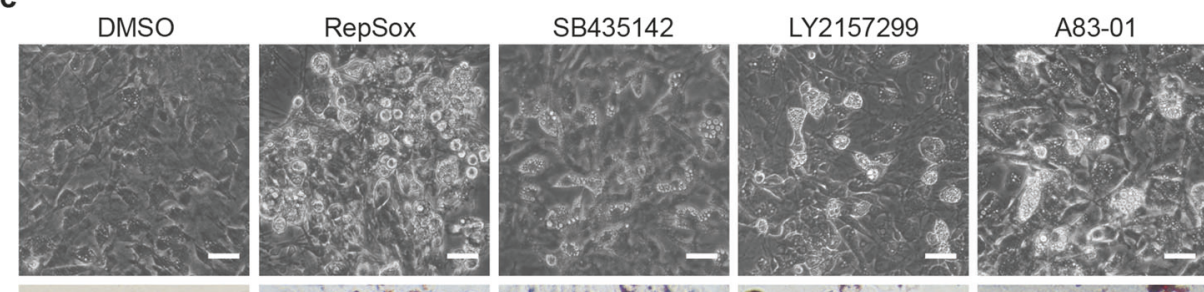

Tranilast
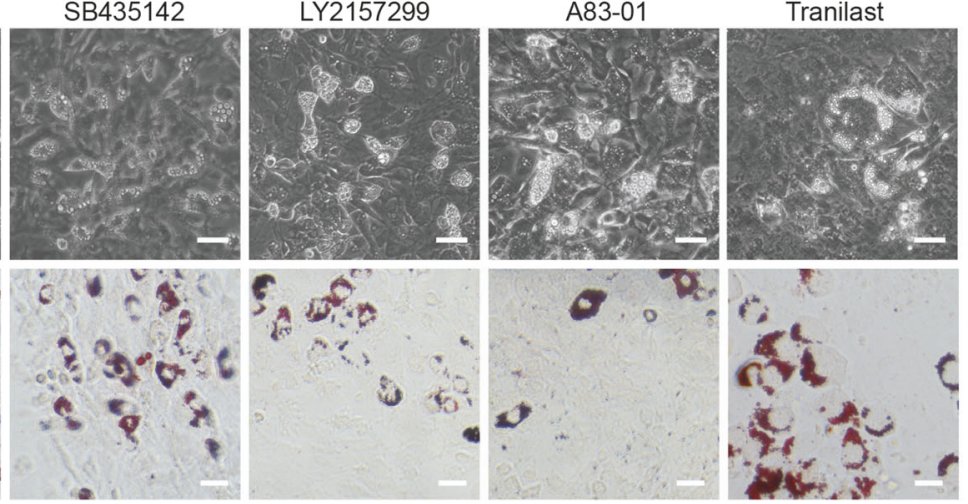

Fig. 1 Inhibitors of the TGF $\beta$ pathway induce the generation of adipocyte-like cells from MEFs. a Phase contrast image of adipocyte-like cells generated from MEFs by treating MEFs with a small-molecule cocktail (10 $\mu \mathrm{M}$ CHIR99021, $10 \mu \mathrm{M}$ RepSox, and $50 \mu \mathrm{M}$ Forskolin) for 10 days. Scale bars, $20 \mu \mathrm{m}$. b Phase contrast (upper panel) and oil red O staining (lower panel) images of MEFs treated with $10 \mu \mathrm{M}$ CHIR99021, $10 \mu \mathrm{M}$ RepSox, or $50 \mu \mathrm{M}$ Forskolin for 10 days. Scale bars, $20 \mu \mathrm{m}$. c Phase contrast (upper panel) and oil red O staining (lower panel) images of MEFs treated with various small-molecule inhibitors of the TGF $\beta$ pathway, including $10 \mu$ M RepSox, $1 \mu$ M SB435142, $1 \mu$ M LY2157299, $10 \mu \mathrm{M}$ Tranilast, and $10 \mu \mathrm{M}$ A83-01, for 10 days. Scale bars, $20 \mu \mathrm{m}$

enhanced adipogenesis (Fig. 4a, b). The adipogenesis-related genes Ppary, C/ebpa, and Ap2, the brown adipocyte markers Ucp1, Prdm16, Pgc1a, Coxiv, and C/ebp $\beta$, and the FAO-related genes Mcad, Lcad, and Cpt2 were all upregulated by RepSox or rosiglitazone, and the combination of both compounds had the best effect (Fig. 4c). Western blot analysis also confirmed the increased UCP1 protein levels in all groups (Fig. 4d). RepSox also increased the number of mitochondria and the protein level of UCP1 (Fig. 4e-g) and enhanced basal, uncoupled, and maximal respiration in cells that differentiated from white preadipocytes (Fig. 4h). These results suggest that RepSox could also induce browning of white preadipocytes.

\section{DISCUSSION}

In this paper, we present a small-molecule strategy to directly reprogram mouse fibroblasts into adipocytes, and the resulting adipocytes possess brown fat characteristics. Although scientists have been able to induce adipocytes from fibroblasts by the combination of growth factors and small-molecule compounds, typically including insulin, IBMX, and dexamethasone (MDI medium) [24], and some compounds have been found to facilitate adipogenesis in combination with the MDI medium [9-13], our study shows for the first time that RepSox alone in fibroblast growth medium (without MDI) is sufficient to induce adipogenesis in MEFs. The adipocytes induced by RepSox express not only genes related to general adipogenesis but also genes specifically associated with brown adipogenesis. The induced cells have the characteristics of brown adipocytes, contain abundant mitochondria and mitochondrial membrane protein UCP1 and have higher levels of mitochondrial respiratory activity. The PPARy agonist rosiglitazone has been reported to promote adipogenesis and the browning of white adipocytes in combination with other adipogenesis inducers. However, adding rosiglitazone alone in fibroblast growth medium could not induce adipocytes from MEFs, which suggests that RepSox might be a stronger inducer than rosiglitazone for brown adipogenesis.

Brown adipocytes are derived from myogenic factor $5\left(\mathrm{Myf5}^{+}\right)$, paired box $7\left(\mathrm{Pax}^{+}\right)$, and engrailed $1\left(E n 1^{+}\right)$multipotent progenitor cells. Early B-cell factor 2 (Ebf2) then regulates the brown preadipocyte lineage specification of the progenitor cells. Finally, PRDM16, a transcription factor that is highly and selectively expressed in brown fat, drives brown adipocyte differentiation through interactions with PPAR $\gamma$, PGC1a, C/EBPs, and other transcription factors [25].

Several signaling pathways have been reported to be involved in regulating the development of BAT. $\beta$-Adrenergic receptor $(\beta-A R)$ signaling is a dominant activating pathway for the development and thermogenesis of brown adipocytes. The activation of $\beta$-ARs by norepinephrine released from the sympathetic nerve terminals increases intercellular CAMP levels, leading to the phosphorylation of protein kinase A (PKA) and p38 mitogen-activated protein kinase (MAPK), which in turn triggers the expression of C/EBPs and PGC1a [26]. This process partially explains our finding that forskolin has weak adipogenesisinducing function, since forskolin activates adenylate cyclase and increases intracellular cAMP levels. In contrast, Wnt ligands such as Wnt10b suppress brown adipose differentiation and 
a

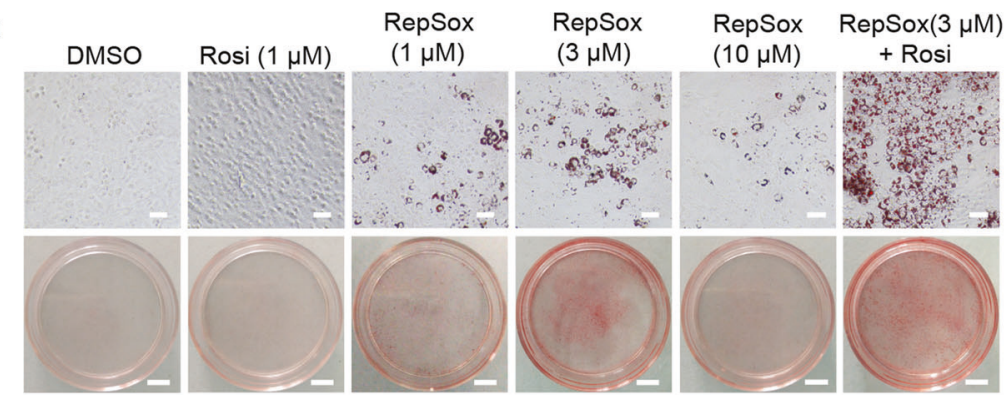

c

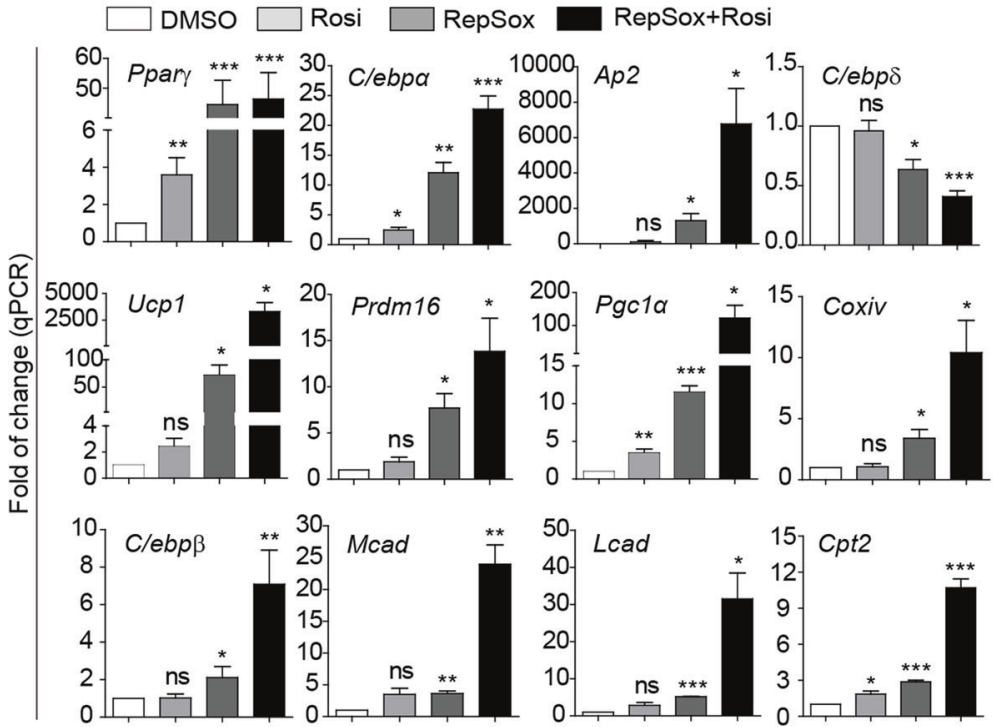

b

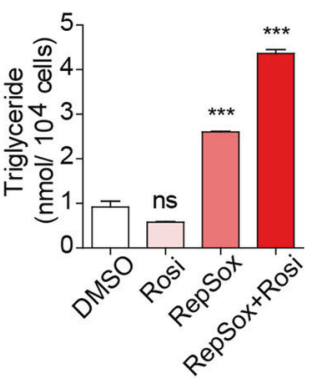

d

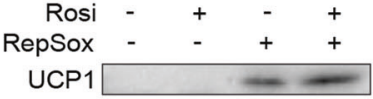

$\beta$-tubulin

f

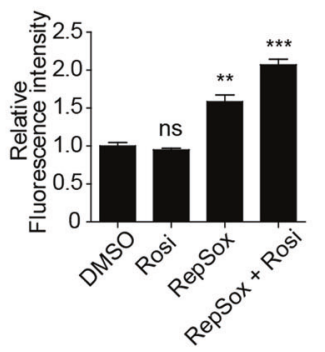

g

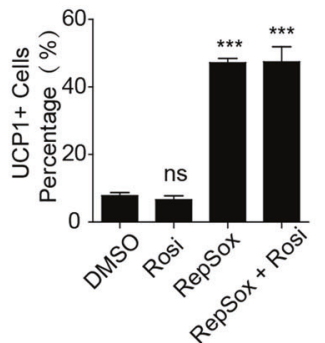

h
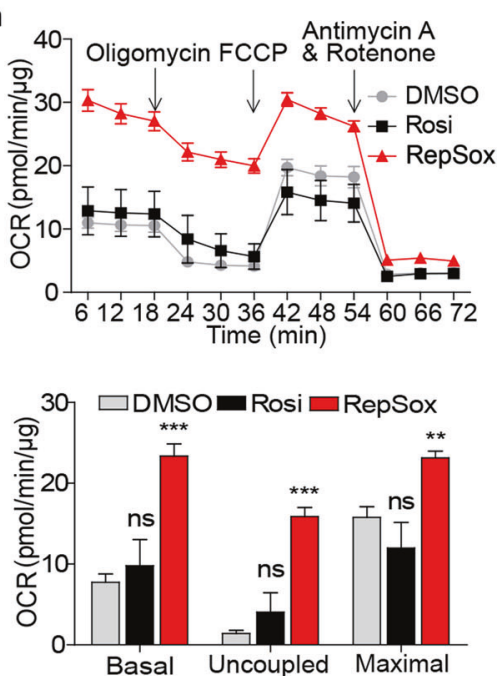

e

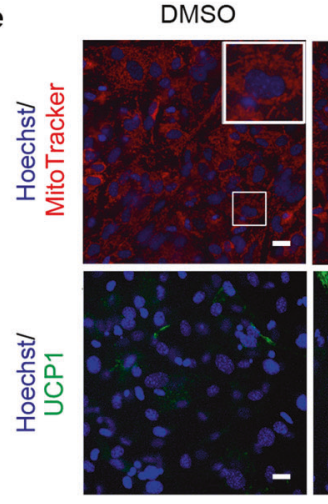

Rosi

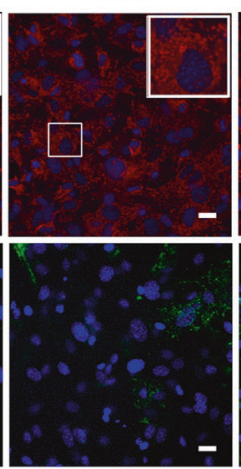

RepSox

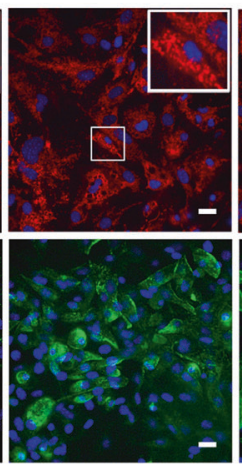

RepSox + Rosi

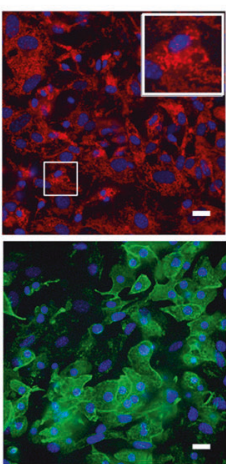

Fig. 2 Characterization of adipogenesis induced by RepSox in MEFs. a Oil red O staining images of MEFs treated with RepSox, rosiglitazone, or their combination for 8 days. Scale bars, $50 \mu \mathrm{m}$ in upper panel and $5 \mathrm{~mm}$ in lower panel. b Statistical analysis of triglyceride concentrations in induced cells $(n=3)$. c qRT-PCR analysis of the mRNA levels of adipogenesis, brown adipocytes, and FAO-related genes in MEFs treated with RepSox $(3 \mu \mathrm{M})$, rosiglitazone $(1 \mu \mathrm{M})$, or their combination for 8 days $(n=3)$. $\mathbf{d}$ Western blot analysis of UCP1 protein levels in induced cells. e Representative images of MitoTracker deep red staining (upper) and immunofluorescence staining of UCP1 (lower) in MEFs treated with $1 \mu \mathrm{M}$ rosiglitazone, $3 \mu \mathrm{M}$ RepSox, or their combination. DMSO was used as a control. Scale bars, $20 \mu \mathrm{m}$. f, $\mathbf{g}$ Statistical analysis of MitoTracker and UCP1 staining presented in e. MitoTracker Deep Red fluorescence intensity is calculated by total fluorescence intensity/image region area $(n=4)$. The percentage of UCP1-positive cells was calculated by the UCP1-positive cell number/total cell number $(n=5)$. $\mathbf{h}$ Measurement of mitochondrial respiration levels and statistics for the basal, uncoupled, and maximal respiration rates of induced cells $(n=5)$. Data are presented as the mean \pm SEM. ${ }^{*} P<0.05 ;{ }^{*} P<0.01 ;{ }^{* *} P<0.001$ 


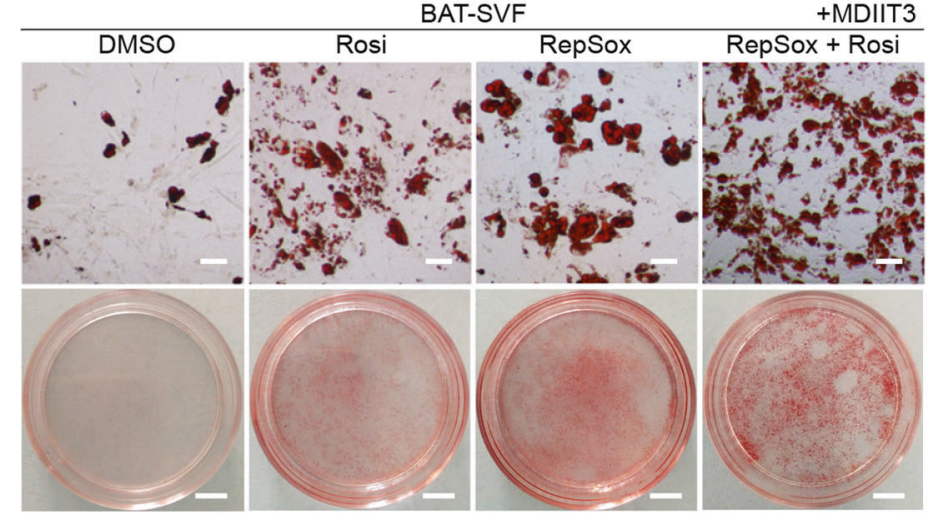

c

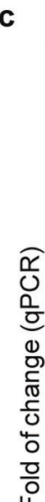

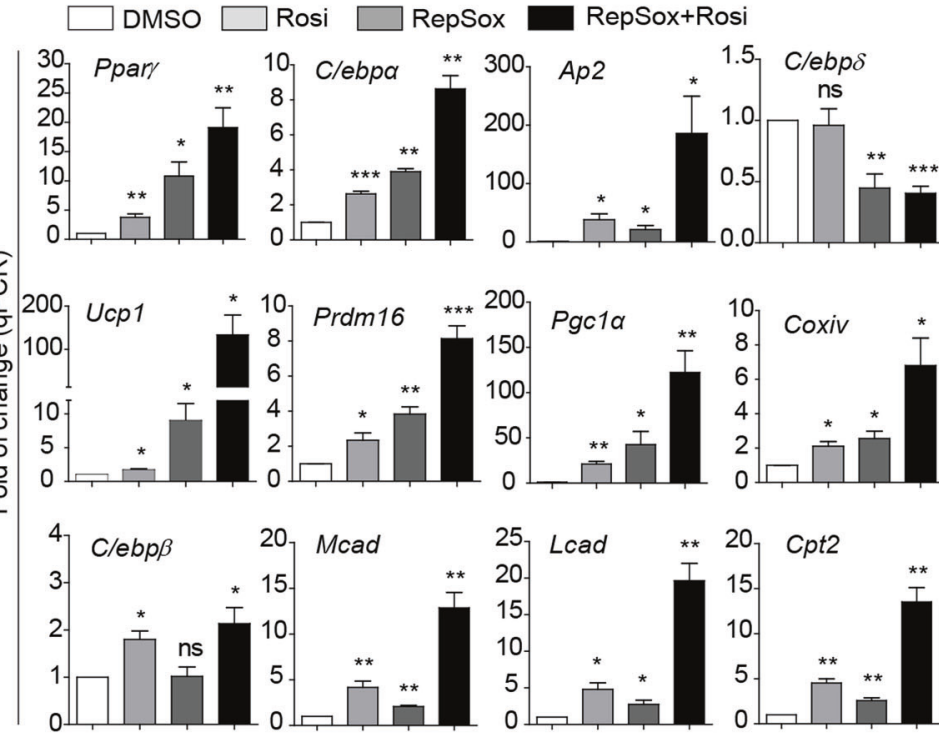

b

d
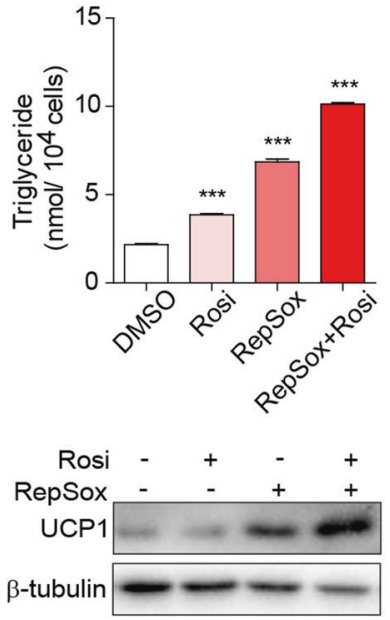

$\mathbf{f}$

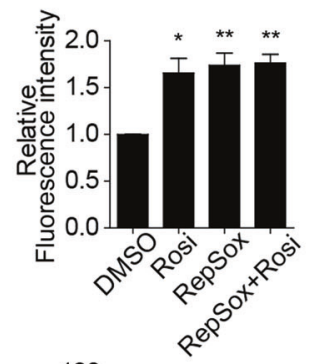

g

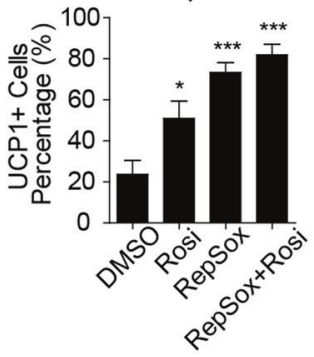

h
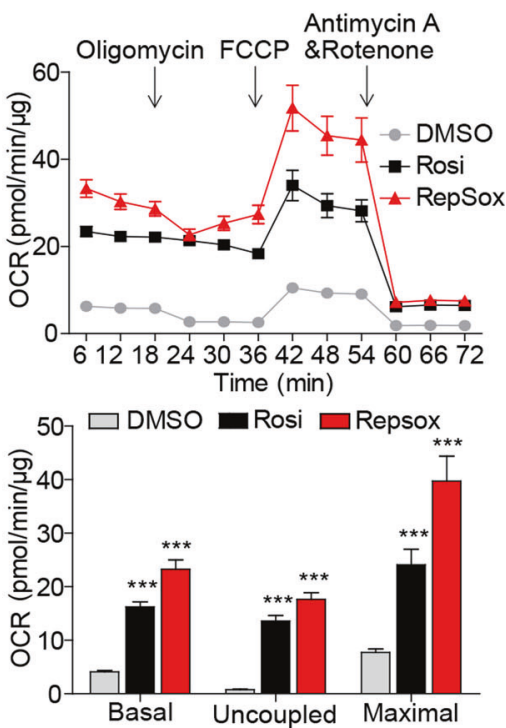

Fig. 3 RepSox enhances differentiation of brown preadipocytes. a Oil red O staining of BAT-SVF (the stromal vascular fraction (SVF) from interscapular BAT, which contains brown preadipocytes) treated with differentiation medium containing $1 \mu \mathrm{M}$ rosiglitazone, $3 \mu \mathrm{M}$ RepSox, or their combination for 8 days. Scale bars, $50 \mu \mathrm{m}$ in upper panel and $5 \mathrm{~mm}$ in lower panel. b Statistical analysis of triglyceride concentrations in induced cells $(n=3)$. c qRT-PCR analysis of the mRNA levels of adipogenesis, brown adipocytes, and FAO-related genes in adipocytes differentiated from BAT-SVF $(n=3)$. d Western blot analysis of UCP1 protein levels in induced cells. e MitoTracker deep red staining (upper) and immunofluorescence staining of UCP1 (lower) in adipocytes differentiated from BAT-SVF under the indicated conditions. Scale bars, $20 \mu \mathrm{m}$. $\mathbf{f}, \mathbf{g}$ Statistical analysis of MitoTracker and UCP1 staining presented in $\mathbf{e}(n=4)$. $\mathbf{h}$ Measurement of mitochondrial respiration levels and statistics for the basal, uncoupled, and maximal respiration rates of induced cells $(n=5)$. Data are presented as the mean \pm SEM. ${ }^{*} P<0.05 ;{ }^{* *} P<0.01 ;{ }^{* * *} P<0.001$ 
a

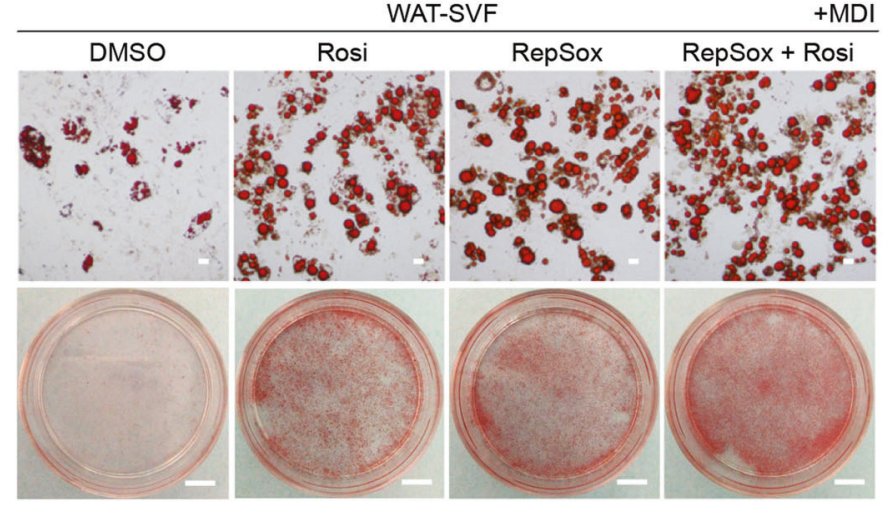

c
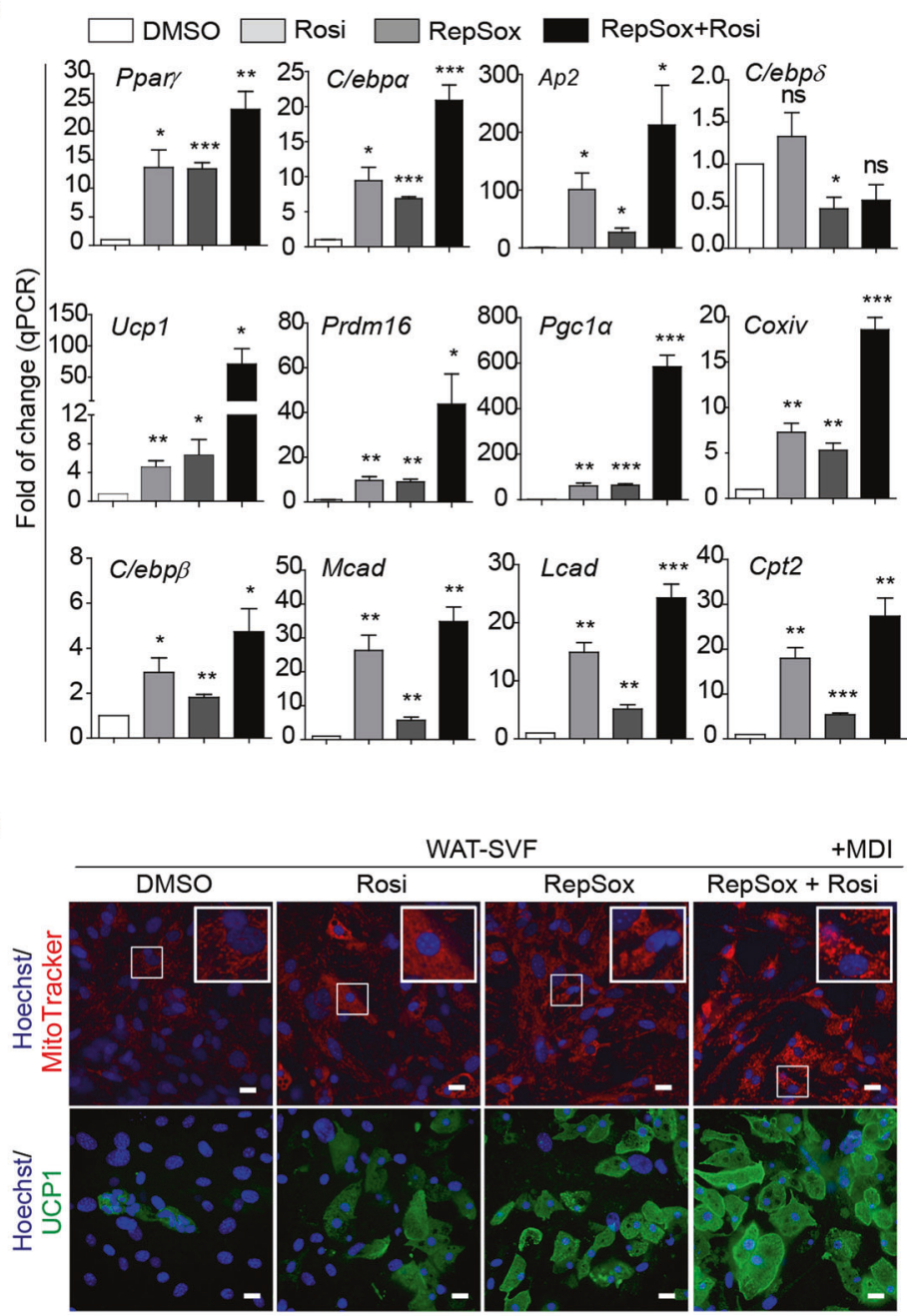

b

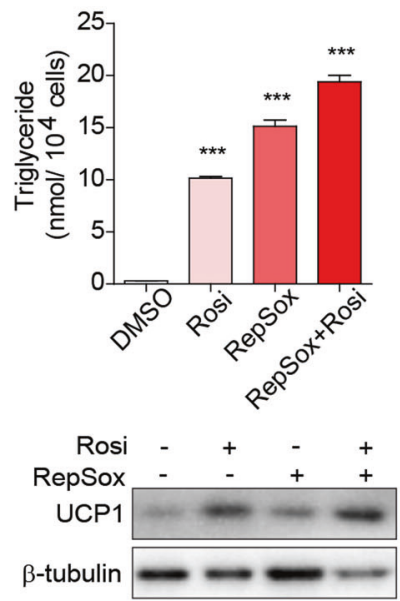

f

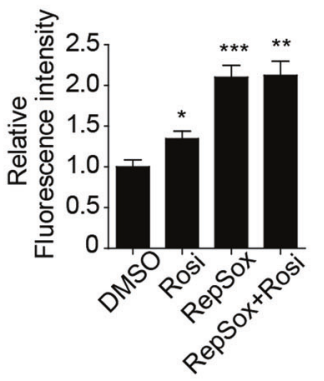

g

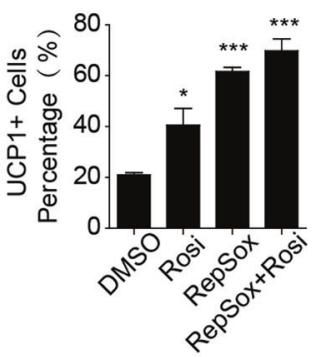

h
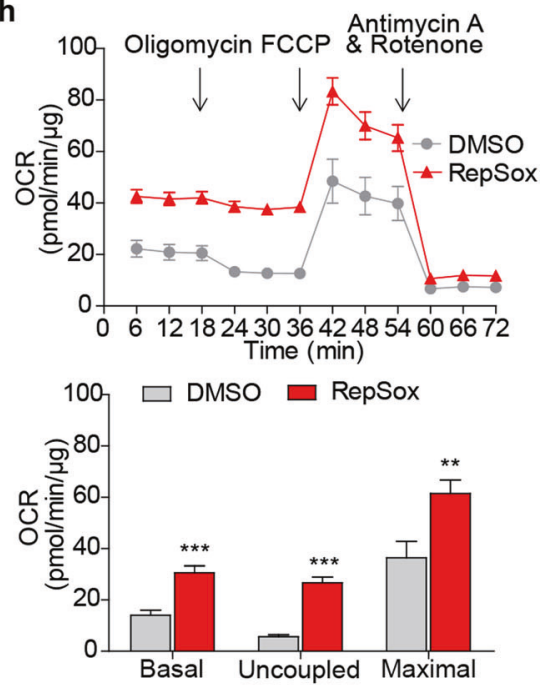

Fig. 4 RepSox promotes browning of white preadipocytes. a Oil red O staining of WAT-SVF (the SVF from subcutaneous WAT, which contains white preadipocytes) treated with differentiation medium containing $1 \mu \mathrm{M}$ rosiglitazone, $3 \mu \mathrm{M}$ RepSox, or their combination for 8 days. Scale bars, $20 \mu \mathrm{m}$ in upper panel and $5 \mathrm{~mm}$ in lower panel. b Statistical analysis of triglyceride concentrations in induced cells $(n=3)$. c qRT-PCR analysis of the mRNA levels of adipogenesis, brown adipocytes, and FAO-related genes in adipocytes differentiated from white preadipocytes $(n=3)$. d Western blot analysis of UCP1 protein levels in induced cells. e MitoTracker deep red staining (upper) and immunofluorescence staining of UCP1 (lower) in adipocytes differentiated from WAT-SVF under the indicated conditions. Scale bars, $20 \mu \mathrm{m}$. $\mathbf{f}, \mathbf{g}$ Statistical analysis of MitoTracker and UCP1 staining presented in $\mathbf{e}(n=4)$. $\mathbf{h}$ Measurement of mitochondrial respiration levels and statistics for the basal, uncoupled, and maximal respiration rates of induced cells $(n=5)$. Data are presented as the mean \pm SEM. ${ }^{*} P<0.05 ;{ }^{* *} P<0.01 ;{ }^{* * *} P<0.001$ 
thermogenesis by repression of PGC1a and UCP1 and can even lead to whitening of mature brown adipocytes. Antagonism of the Wnt pathway activates PPARy and promotes the differentiation of fat precursor cells into brown adipocytes [27]. The mTOR signaling pathway also plays an important role in the differentiation of adipocytes. The inhibition of $\mathrm{mTORC} 1$ is required for the induction of WAT browning under low temperature in vivo [28]. Here, we demonstrate that blocking TGF- $\beta$ signaling also promotes brown adipogenesis.

TGF- $\beta$ signaling is involved in many cellular processes. The binding of ligands triggers the assembly of type I and type II receptors into heterogeneous complexes. Type II receptors phosphorylate and activate type I receptors. The signals are then transferred from the receptor-regulated Smads (R-Smads) to the common-Smad (co-Smad), Smad4, and finally into the nucleus to modulate the transcription of target genes [29]. The vertebrate TGF $\beta$ superfamily contains more than 30 ligands, including TGF $\beta$ s, BMPs, growth differentiation factors (GDFs), activins, nodal, and others [30]. Each member of the TGF- $\beta$ family has its own combination of type I and type II receptors and R-Smads. For BMP signaling, the type I receptors ALK $1 / 2 / 3 / 6$ and the type II receptor BMPRII are involved in the activation of Smad1/5/8 [31]. For TGF- $\beta$ (including TGF- $\beta 1 / 2 / 3$ ) signaling, the type I receptor T $\beta R I / A L K 5$ and the type II receptor T $\beta R$ II activate Smad2/3 [18]. For activin/ nodal signaling, the type I receptors ALK4/7 also activate Smad2/3 with the type II receptors ActRII and ActRIIB [32].

The functions of TGF- $\beta$ signaling in adipogenesis are complicated. BMP4 and BMP7 have been reported to induce brown adipogenic regulators in fibroblasts, mesenchymal stem cells, or adipogenic precursor cells $[9,33]$. Although BMP8b does not affect the differentiation of brown adipocytes, it positively regulates the thermogenesis of mature brown adipocytes [34]. In contrast, some other TGF- $\beta$ members, including TGF- $\beta 1$, activins, and GDF- 8 (myostatin), negatively regulate brown fat differentiation and BAT thermogenesis [26]. Genetic deletion of Smad3, a major downstream factor of TGF- $\beta$ signaling, promotes mitochondrial functions and induces browning of white fat in mice [35]. Treatment with anti-TGF- $\beta$ neutralization antibody also improves glucose tolerance and energy homeostasis and elevates expression of BATspecific proteins in obese mice [35].

RepSox is a selective TGF $\beta$ RI/ALK5 inhibitor that inhibits the binding of ATP to ALK5 and subsequent ALK5 phosphorylation, thus inhibiting TGF- $\beta$ signaling $[14,15]$. Our results show that the treatment of RepSox induces brown adipogenesis from primary mouse fibroblasts and fat precursor cells, which is consistent with previous reports that some TGF- $\beta$ members, especially TGF- $\beta 1$, negatively regulate brown fat differentiation [26]. In addition to RepSox, we also investigated other inhibitors of the TGF- $\beta$ signaling pathway in adipogenesis. SB431542 and A83-01 inhibit TGFBRI/ALK5 and the type I receptor ALK4/7 [17, 19]. LY2157299 is an inhibitor of the TGF $\beta R I / A L K 5$ kinase that specifically downregulates the phosphorylation of Smad2 [18]. On the other hand, Tranilast inhibits the release of TGF $\beta 1$ and TGF $\beta 2$ [20]. All these compounds have an effect similar to RepSox in inducing adipogenesis in MEFs.

In conclusion, this study demonstrates that one small-molecule compound, RepSox, an inhibitor of the TGF- $\beta$ receptor, is sufficient to induce brown adipogenesis in mouse fibroblasts and fat precursor cells. Combined with previous research, these data suggest that inhibitors of the TGF- $\beta$ signaling pathway might be developed as new therapeutics for obesity and type 2 diabetes.

\section{ACKNOWLEDGEMENTS}

This work was supported by grants from the Ministry of Science and Technology of China (Nos. 2015CB964503 and 2017YFA0104002), the Chinese Academy of Sciences (No. XDA16010202), and the National Natural Science Foundation of China (Nos. 81425024,81730099 , and 81472862).

\section{AUTHOR CONTRIBUTIONS}

WZT, YBF, and $X X$ designed the research; WZT and YBF performed the research; WZT, YBF, and XX analyzed the data and wrote the paper.

\section{ADDITIONAL INFORMATION}

Competing interests: The authors declare no conflict of interest.

\section{REFERENCES}

1. Barnett R. Obesity. Lancet. 2005;365:1843.

2. Rosen ED, Spiegelman BM. What we talk about when we talk about fat. Cell. 2014;156:20-44.

3. Fedorenko A, Lishko PV, Kirichok Y. Mechanism of fatty-acid-dependent UCP1 uncoupling in brown fat mitochondria. Cell. 2012;151:400-13.

4. Cohen $P$, Spiegelman BM. Brown and beige fat: molecular parts of a thermogenic machine. Diabetes. 2015;64:2346-51.

5. Vitali A, Murano I, Zingaretti MC, Frontini A, Ricquier D, Cinti S. The adipose organ of obesity-prone C57BL/6J mice is composed of mixed white and brown adipocytes. J Lipid Res. 2012;53:619-29.

6. Van Marken Lichtenbelt WD, Vanhommerig JW, Smulders NM, Drossaerts JM, Kemerink GJ, Bouvy ND, et al. Cold-activated brown adipose tissue in healthy men. N Engl J Med. 2009;360:1500-8.

7. Sidossis LS, Porter C, Saraf MK, Borsheim E, Radhakrishnan RS, Chao T, et al. Browning of subcutaneous white adipose tissue in humans after severe adrenergic stress. Cell Metab. 2015;22:219-27.

8. Harms M, Seale P. Brown and beige fat: development, function and therapeutic potential. Nat Med. 2013;19:1252-63.

9. Tseng Y, Kokkotou E, Schulz TJ, Huang TL, Winnay JN, Taniguchi CM, et al. New role of bone morphogenetic protein 7 in brown adipogenesis and energy expenditure. Nature. 2008;454:1000-4.

10. Zhang Y, Li R, Meng Y, Li SW, Donelan W, Zhao Y, et al. Irisin stimulates browning of white adipocytes through mitogen-activated protein kinase p38 MAP Kinase and ERK MAP kinase signaling. Diabetes. 2014;63:514-25.

11. Ohno $H$, Shinoda $K$, Spiegelman Bruce $M$, Kajimura S. PPAR $\gamma$ agonists induce a white-to-brown fat conversion through stabilization of PRDM16 protein. Cell Metab. 2012;15:395-404.

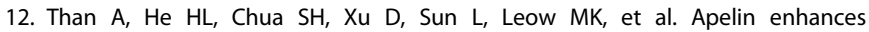
brown adipogenesis and browning of white adipocytes. J Biol Chem. 2015;290: 14679-91.

13. Moisan A, Lee YK, Zhang JD, Hudak CS, Meyer CA, Prummer M, et al. White-tobrown metabolic conversion of human adipocytes by JAK inhibition. Nat Cell Biol. 2015;17:57-67.

14. Gellibert F, Woolven J, Fouchet MH, Mathews N, Goodland H, Lovegrove V, et al. Identification of 1,5-Naphthyridine derivatives as a novel series of potent and selective TGF- $\beta$ type I receptor inhibitors. J Med Chem. 2004;47: 4494-506.

15. Ichida JK, Blanchard J, Lam K, Son EY, Chung JE, Egli D, et al. A small-molecule inhibitor of Tgf- $\beta$ signaling replaces Sox 2 in reprogramming by inducing Nanog. Cell Stem Cell. 2009;5:491-503.

16. Fu $Y$, Huang $C, X u X, G u H, Y e ~ Y$, Jiang $C$, et al. Direct reprogramming of mouse fibroblasts into cardiomyocytes with chemical cocktails. Cell Res. 2015;25: 1013-24.

17. Gareth Jl, James FC, John DH, Laramie MG, Alastair DR, Caroline SH. SB-431542 is a potent and specific inhibitor of transforming growth factor- $\beta$ superfamily type I activin receptor-like kinase (ALK) receptors ALK4, ALK5, and ALK7. Mol Phys. 2002;62:65-74.

18. Herbertz S, Sawyer JS, Stauber AJ, Gueorguieva I, Driscoll KE, Estrem ST, et al. Clinical development of galunisertib (LY2157299 monohydrate), a small molecule inhibitor of transforming growth factor-beta signaling pathway. Drug Des Dev Ther. 2015;9:4479-99.

19. Tojo $M$, Hamashima $Y$, Hanyu A, Kajimoto T, Saitoh M, Miyazono $K$, et al. The ALK5 inhibitor A-83-01 inhibits Smad signaling and epithelial-to-mesenchymal transition by transforming growth factor- $\beta$. Cancer Sci. 2005;96:791-800.

20. Platten M, Wild-Bode C, Wick W, Leitlein J, Dichgans J, Weller M. N-[3,4-dimethoxycinnamoyl]-anthranilic acid (tranilast) inhibits transforming growth factor- $\beta$ release and reduces migration and invasiveness of human malignant glioma cells. Int J Cancer. 2001;93:53-61.

21. Farmer SR. Transcriptional control of adipocyte formation. Cell Metab. 2006;4: 263-73.

22. Cao ZD, Umek RM, McKnight SL. Regulated expression of three C/EBP isoforms during adipose conversion of 3T3-L1 cells. Gene Dev. 1991;5:1538-52.

23. Aune UL, Ruiz L, Kajimura S. Isolation and differentiation of stromal vascular cells to beige/brite cells. J Vis Exp. 2013;73:1-6. 
24. Klemm DJ, Leitner JW, Watson P, Nesterova A, Reusch JE, Goalstone ML, et al. Insulin-induced adipocyte differentiation. Activation of CREB rescues adipogenesis from the arrest caused by inhibition of prenylation. J Biol Chem. 2001;276:28430-5.

25. Wang W, Seale P. Control of brown and beige fat development. Nat Rev Mol Cell Biol. 2016;17:691-702

26. Kajimura S, Saito M. A new era in brown adipose tissue biology: molecular control of brown fat development and energy homeostasis. Annu Rev Physiol. 2014;76: 225-49.

27. Kang S, Bajnok L, Longo KA, Petersen RK, Hansen JB, Kristiansen K, et al. Effects of Wnt signaling on brown adipocyte differentiation and metabolism mediated by PGC-1a. Mol Cell Biol. 2005;25:1272-82.

28. Liu M, Bai J, He S, Villarreal R, Hu D, Zhang C, et al. Grb10 promotes lipolysis and thermogenesis by phosphorylation-dependent feedback inhibition of mTORC1. Cell Metab. 2014;19:967-80.

29. Hata A, Chen YG. TGF- $\beta$ signaling from receptors to Smads. Cold Spring Harb Perspect Biol. 2016;8:1-31.
30. Schmierer B, Hill CS. TGF $\beta-S M A D$ signal transduction: molecular specificity and functional flexibility. Nat Rev Mol Cell Biol. 2007;8:970-82.

31. Sieber C, Kopf J, Hiepen C, Knaus P. Recent advances in BMP receptor signaling Cytokine Growth Factor Rev. 2009;20:343-55.

32. Massague J. TGF $\beta$ signalling in context. Nat Rev Mol Cell Biol. 2012;13: 616-30.

33. Qian SW, Tang Y, Li X, Liu Y, Zhang YY, Huang HY, et al. BMP4-mediated brown fat-like changes in white adipose tissue alter glucose and energy homeostasis. Proc Natl Acad Sci USA. 2013;110:798-807.

34. Whittle AJ, Carobbio S, Martins L, Slawik M, Hondares E, Vazquez MJ, et al. BMP8B increases brown adipose tissue thermogenesis through both central and peripheral actions. Cell. 2012;149:871-85.

35. Yadav H, Quijano C, Kamaraju Anil K, Gavrilova O, Malek R, Chen W, et al Protection from obesity and diabetes by blockade of TGF- $\beta /$ Smad3 signaling. Cell Metab. 2011;14:67-79. 